\title{
Near-simultaneous magnetotail flux rope observations with Cluster and Double Star
}

\author{
A. P. Walsh ${ }^{1}$, A. N. Fazakerley ${ }^{1}$, R. J. Wilson ${ }^{1,2}$, I. V. Alexeev ${ }^{1}$, P. D. Henderson ${ }^{1}$, C. J. Owen ${ }^{1}$, E. Lucek ${ }^{3}$, C. Carr ${ }^{3}$, \\ and I. Dandouras ${ }^{4}$ \\ ${ }^{1}$ Mullard Space Science Laboratory, University College London, Holmbury St. Mary, Dorking, RH5 6NT, UK \\ ${ }^{2}$ Los Alamos National Laboratory, Los Alamos, New Mexico, USA \\ ${ }^{3}$ Space and Atmospheric Physics, Imperial College London, SW7 2BZ, UK \\ ${ }^{4}$ Centre d'Etude Spatiale des Rayonnements, Toulouse, France
}

Received: 15 January 2007 - Revised: 16 July 2007 - Accepted: 9 August 2007 - Published: 29 August 2007

\begin{abstract}
We present observations of three magnetic flux ropes in the tail of the Earth's magnetosphere on $7 \mathrm{Au}-$ gust 2004 by the Cluster and Double Star TC-1 spacecraft. The first two flux rope signatures were observed, nearsimultaneously, by Cluster and TC-1, which were located at $(-16.3,-8.7,0.10) R_{E}$ GSM and $(-10.3,-7.11,0.81) R_{E}$ GSM, respectively, a separation of $6.3 R_{E}$. A third signature was observed some four minutes later by two of the four Cluster spacecraft, while the other two spacecraft observed a feature resembling a Travelling Compression Region (TCR). These observations are interpreted as three individual flux ropes existing in the magnetotail, the first two, at least, simultaneously. The formation mechanism of the flux ropes and the consequences of their presence for the structure of the magnetotail on this day are discussed in the context of multiple X-point reconnection.
\end{abstract}

Keywords. Magnetosphere (magnetotail; plasma sheet, storms and substorms)

\section{Introduction}

A widely invoked substorm model, the Near-Earth Neutral Line (NENL) Model (e.g. Baker et al., 1996), involves reconnection at a neutral point in the near-Earth tail. However this model does not emphasize the possibility of reconnection at more than one site in the tail, or so-called multiple X-point reconnection (MXR). If they exist, multiple X-points need not be reconnecting at the same rate (Schindler, 1974). Each of the X-lines would first have to reconnect closed, plasma sheet field lines before reaching the open field lines of the lobes. The fastest reconnecting X-line would thus begin to reconnect open lobe field lines before the others, resulting in the fast jets that are characteristic of the reconnection of

Correspondence to: A. P. Walsh

(apw@mssl.ucl.ac.uk) lobe field lines. The remaining $\mathrm{X}$-lines in the system, and the loops of magnetic flux in between them, would then be swept Earthward or tailward depending on their location with respect to the fastest, dominant, $\mathrm{X}$-line. If there is a cross-tail component of the magnetic field present in the magnetotail, the reconnected flux that lies between the X-lines is expected to form helical structures of magnetic flux, or magnetic flux ropes (Hughes and Sibeck, 1987). The simplest model of the flux rope is known as the force-free flux rope (e.g. Lepping et al., 1990), which is the minimum energy configuration for helical magnetic fields. The force-free model is so-called because currents in the flux rope run parallel to the direction of the local magnetic field, i.e. the magnetic component of the Lorentz force, $\boldsymbol{J} \times \boldsymbol{B}$, is equal to zero. The structure of the ideal force-free flux rope is cylindrical with an azimuthal magnetic field at the edge of the rope which becomes increasingly directed along the axis of the rope toward its center. Flux ropes, similar to those described by a force-free model, have been observed by Geotail, moving both Earthward, embedded in bursty bulk flows, and tailward, as socalled plasmoid type flux ropes (Slavin et al., 2003a). Earthward moving flux ropes have also been observed by Cluster (e.g. Slavin et al., 2003b; Henderson et al., 2006), which implies that multiple X-lines may exist in the tail within $20 R_{E}$ of the Earth.

There is a theoretical basis for MXR (Zwingmann, 1983), and its relation to flux ropes (Ogino et al., 1990), however observational evidence is often based on measurements taken either by a single spacecraft or at a single location in the tail (e.g. Eastwood et al., 2005). Observations of structures related to MXR, for example flux ropes, existing simultaneously at different locations in the tail would provide valuable experimental evidence for the theory. In this paper we present unusual observations from the Cluster and Double Star TC-1 spacecraft of two flux ropes that we interpret as existing simultaneously in the tail, separated by approximately $6 R_{E}$, and a third flux rope observed by Cluster some $4 \mathrm{~min}$

Published by Copernicus Publications on behalf of the European Geosciences Union. 
later. To the best of our knowledge, these are the first such observations.

\section{Observations}

\subsection{Event context}

Between 7 August 2004, 19:00 UT and 8 August 2004, 00:30 UT, the Auroral Electrojet (AE) index (not shown here) shows four small enhancements, indicating increased magnetospheric convection, at $\sim 18: 50 \mathrm{UT}, \sim 20: 05 \mathrm{UT}, \sim 21: 20 \mathrm{UT}$ and $\sim 23: 00$ UT. During this interval the Cluster tetrahedron and Double Star TC-1 were approximately conjugate in the post-midnight sector of the Earth's magnetotail. We will concentrate on the fourth $\mathrm{AE}$ enhancement in this period, thought by Nakamura et al. (2005) to be related to a substorm with an onset at 23:00 UT. IMAGE-FUV (Mende et al., 2000) observations (not shown here) reveal two bright spots in the aurora at 23:00 UT, separated by $<5^{\circ}$ magnetic latitude and $2 \mathrm{~h}$ MLT, while onset of the auroral substorm occurs at 23:09 UT (Frey and Mende, 2006). During this interval the four Cluster spacecraft (henceforth referred to as $\mathrm{C} 1$, $\mathrm{C} 2, \mathrm{C} 3$ and $\mathrm{C} 4)$, configured in an approximately equilateral tetrahedron of side $\sim 1000 \mathrm{~km}$, were located at $(-16.3,-8.7$, $0.1) R_{E}$ GSM. Double Star TC-1 was located at $(-10.3,-7.1$, $0.8) R_{E}$ GSM, a separation of $6.3 R_{E}$ from Cluster (GSM coordinates will be used throughout this paper, unless otherwise specified). At this time the Geotail spacecraft was located at (17.2, 6.0, 3.9) $R_{E}$, upstream of the Earth, providing a solar wind monitor. The IMF (not shown) was directed predominantly southward from 21:00-22:00 UT, turning northward at 22:00 UT and remaining that way until the end of the interval. The IMF was, however, dominated by a positive $B_{y}$ until 22:45 UT at which point a change in $B_{z}$ caused the clock angle to rotate from $\sim 80^{\circ}$ to $\sim 40^{\circ}$. At 23:20 UT the clock angle returned to $\sim 80^{\circ}$. The presence of a $B_{y}$-dominated IMF might have caused a twisting of the tail along its axis (e.g. Cowley, 1981).

\subsection{Event overview}

In Fig. 1, we give an overview of the interval of interest (22:50 UT-23:50 UT) for Cluster and TC-1. Panel (a) is a PEACE (Johnstone et al., 1997) electron spectrogram from $\mathrm{C} 1$ and panel (b) the equivalent PEACE (Fazakerley et al., 2005) spectrogram from TC-1. Panel (c) shows the plasma$\beta$ from $\mathrm{C} 1$ (protons) and TC-1 (ions). Panels ( $\mathrm{d}-\mathrm{g})$ show the three components and magnitude of the magnetic field from FGM on all four Cluster spacecraft (Balogh et al., 2001) and TC-1 (Carr et al., 2005). Panel (h) shows the $x$ component of velocity perpendicular to the magnetic field, projected onto GSM axes $\left(v_{\perp x}\right)$ from CIS-CODIF (protons) (Rème et al., 2001) for $\mathrm{C} 1$ and $\mathrm{C} 4$ and HIA (ions) (Rème et al., 2005) for TC-1 (the TC-1 velocity has been multiplied by 2 for clarity). This quantity is a proxy for magnetic flux transport.
At 22:50 UT Cluster observed a plasma sheet-like electron population (Fig. 1, panel a) $\beta \sim 5$ (Fig. 1, panel c) and a $B_{x}$ $\sim-10 \mathrm{nT}$ (Fig. 1, panel d), indicating the tetrahedron was located in the central plasma sheet south of the neutral sheet. A short-lived weakening of $B_{x}$ to $\sim-2 \mathrm{nT}$ at 22:58 UT, accompanied by an increase in the plasma $\beta$ to $\sim 10$ indicates that the spacecraft moved closer to the neutral sheet but did not cross it. A neutral sheet crossing occurred at 23:02 UT, indicated by a change of sign in $B_{x}$ from negative to positive, again accompanied by an increase in $\beta$ to $\sim 10$. At 22:50 UT, $v_{\perp}$ was close to zero. Between 22:58 UT to 23:03 UT however, at the time of the neutral sheet encounter and crossing $v_{\perp x}$ strengthened, reaching a value of approximately $300 \mathrm{~km} \mathrm{~s}^{-1}$ (Fig. 1, panel h). This tailward-directed fast flow may indicate the presence of an active reconnection $\mathrm{X}$-line Earthward of Cluster.

Shortly after the neutral sheet crossing at 23:02 UT, the Cluster spacecraft encountered the neutral sheet again. In the period 23:04-23:10 UT C1 and C4 remained predominantly north of the neutral sheet, C3 predominantly south of the neutral sheet while $\mathrm{C} 2$ crossed it three times. This indicates that the current sheet was thinner than, or as thin as, the greatest separation of the Cluster spacecraft. From 23:06 UT to 23:09 UT a stronger tailward fast flow was also measured $\left(v_{\perp x} \sim-700 \mathrm{~km} \mathrm{~s}^{-1}\right)$ and enhanced electron fluxes were seen. At 23:09 UT all four spacecraft exited the thin current sheet region, moving north of the neutral sheet. The plasma $\beta$ dropped below 1 at this point, and remained close to 1 until $\sim 23: 25 \mathrm{UT}$, placing the spacecraft closer to the Plasma Sheet Boundary Layer (PSBL) during this period. Between 23:27 UT and 23:33 UT, the tetrahedron crossed the neutral sheet again,, measuring a plasma with hotter electrons then previously and a high $\beta$. An Earthward-directed fast flow of $\sim 400 \mathrm{~km} \mathrm{~s}^{-1}$ was also detected, now suggesting an active $\mathrm{X}$-line tailward of Cluster.

We will focus on a period beginning at 23:12 UT, when the spacecraft briefly returned to the central plasma sheet $\left(B_{x}\right.$ small) and a short Earthward flow of $v_{\perp x} \sim 200 \mathrm{~km} \mathrm{~s}^{-1}$ was detected, together with a south-north bipolar signature in $B_{z}$ and a peak in $B_{y}$ (Signature 1). A smaller south-north bipolar signature was seen by Cluster at 23:16 UT (Signature 3), accompanied by Earthward flows of $\sim 120 \mathrm{kms}^{-1}$. At both times enhanced electron fluxes were seen.

At 22:50 UT TC-1, like Cluster, was located south of the tail neutral sheet in the plasma sheet. At 22:59 UT, the spacecraft crossed the neutral sheet and remained in the northern plasma sheet until 23:05 UT, after which it crossed the neutral sheet several times before 23:21 UT and then remained predominantly south of the neutral sheet until the end of the interval. HIA observed Earthward-directed flows of $v_{\perp x}$ $\sim 150 \mathrm{~km} \mathrm{~s}^{-1}$ from 23:04 UT to 23:08 UT and 23:10 UT to $23: 14$ UT at which time a flow reversal occurred and $v_{\perp x}$ changed to $\sim-120 \mathrm{~km} \mathrm{~s}^{-1}$. These flows lasted until 23:21 UT. A south-north bipolar signature in $B_{z}$ (Signature 2) was observed at $\sim 23: 12$ UT. Given that the plasma $\beta$ 
remained $\sim 2$ after the initial neutral sheet crossing it can be inferred that TC-1 was located in the central plasma sheet throughout the interval. The presence of plasma sheet-like electrons in the PEACE spectrogram confirms this. Between 23:05 UT and 23:35 UT $B_{z}$ increased steadily from $5 \mathrm{nT}$ to $19 \mathrm{nT}$, the magnetic field near TC-1 becoming increasingly dipolar in character, consistent with reconnection occurring in the tail throughout this interval. However between 23:05 UT and 23:25 UT the $B_{y}$ component decreased by a similar amount, although the magnitude of the magnetic field remained approximately the same. This is consistent with a rotation of the tail about its axis, and may be related to the IMF clock angle rotations observed by Geotail. These Cluster and TC-1 data, have been interpreted by Nakamura et al. (2005) in their study of dipolarizations during this event as indicating the presence of a "source of disturbance" (which we have interpreted as at least one reconnection X-line) located between TC-1 and the Cluster tetrahedron.

\subsection{Flux rope observations}

The south-north bipolar signatures observed in $B_{z}$ by Cluster and TC-1 at 23:12 UT (Signatures 1 and 2, respectively) were accompanied by peaks seen in $B_{y}$ and $|\boldsymbol{B}|$. While bipolar signatures in the magnetic field can be caused by numerous phenomena, the observation of a well-formed core field in these events means that these signatures can be interpreted in terms of a simple flux rope model as an Earthward moving flux rope passing over the spacecraft. This is supported by the observed direction of $\boldsymbol{v}_{\perp}$ at both Cluster and TC-1, and the results of 4-spacecraft timing on the peak in $B_{y}$ by Cluster, which determined the propagation velocity of Signature 1 across the tetrahedron to be $146 \mathrm{~km} \mathrm{~s}^{-1}$ in an Earthward direction, comparable to the ion measured ion flow velocity, $v_{\perp}$. The flux rope signatures and exact times of the encounters at $\mathrm{C} 1$ and TC- 1 are shown in Fig. 2. The time of a flux rope encounter is defined here as the time at which the local maximum in $|\boldsymbol{B}|$ is seen by a spacecraft. The four Cluster spacecraft each encountered the flux rope between 23:12:14 UT (SC3) and 23:12:21 UT (SC4) and TC-1 encountered a flux rope at 23:12:28 UT. Note that bipolar signatures observed in the GSM coordinate frame (Fig. 2, panel c) need not pass through $B_{z}=0$ to represent a flux rope; flux ropes are not necessarily perfectly aligned with a GSM axis. A coordinate frame closer to the natural frame of the flux rope will reveal a true bipolar signature in one of its components. Figure 2, panels (e-j), show the flux rope signatures in such a coordinate system, as determined by minimum variance analysis (MVA) (Sonnerup and Cahill, 1967). In the ideal case, for a force-free flux rope, the bipolar signature is usually found in the maximum variance component and the core field in the intermediate component (Xiao et al., 2004). Similarly to Slavin et al. (2003a), who used a force free flux rope model to refine MVA results, we have also analysed the flux rope signatures with another technique, discussed in

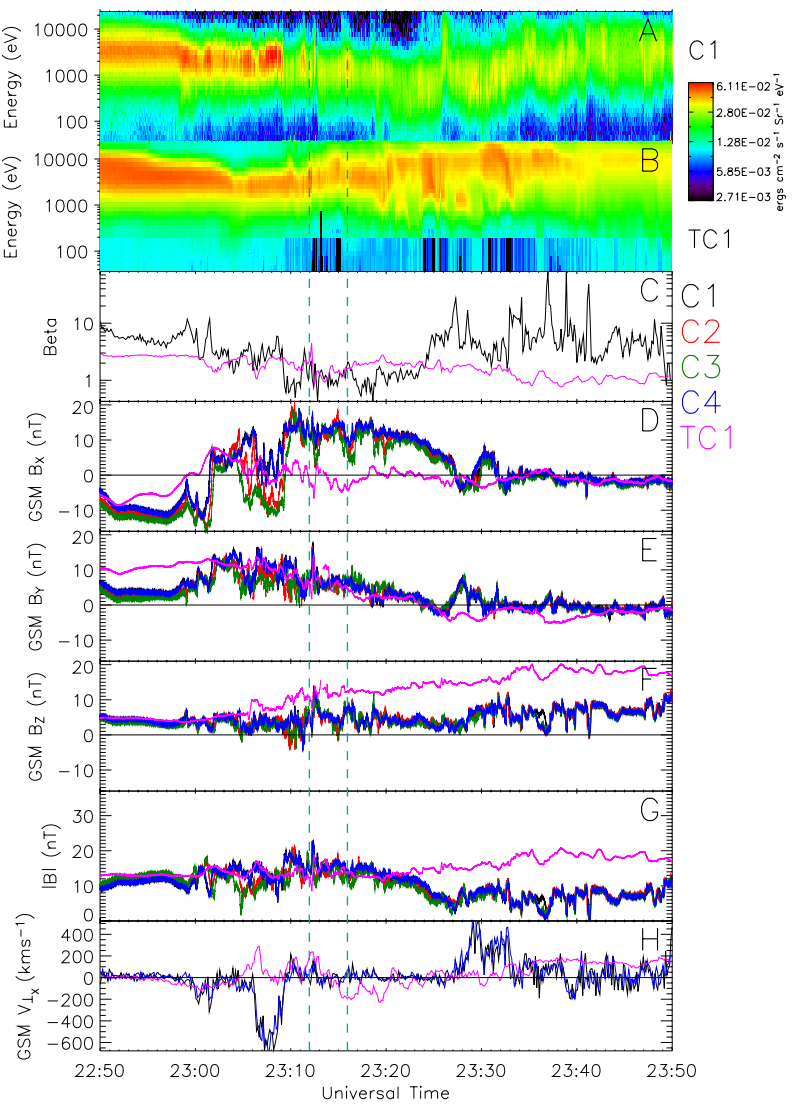

Fig. 1. Overview of the event. Panel (A) is a PEACE electron spectrogram from Cluster 1, Panel (B) the equivalent spectrogram from Double Star TC-1. Panel (C) is the plasma beta for C1 (black) and TC-1 (magenta), calculated from CIS-CODIF and HIA, respectively, and FGM. Panels (D), (E), (F) and (G) show the GSM X, $\mathrm{Y}$ and $\mathrm{Z}$ components and magnitude of the magnetic field, respectively, for all four Cluster spacecraft (C1 - black, C2 - red, C3 green, C4 - blue) and TC-1 (magenta), as measured by the FGM instruments. Panel (H) shows GSM $v_{\perp x}$, the velocity of magnetic flux transport, as measured by CIS-CODIF (C1, black and C4, blue) and HIA (TC-1, magenta). The TC-1 velocity is multiplied by 2 , for clarity. The two vertical dashed lines mark the times of spacecraft encounters with Signatures $1 \& 2$, and Signature 3, respectively.

Sect. 3. The signature seen by Cluster at 23:16:24 UT (Signature 3) was not the same over all four spacecraft. $C 2$ and C3 saw a bipolar signature in GSM $B_{z}$, and peaks in $B_{y}$ and $|\boldsymbol{B}|$, whereas $\mathrm{C} 1$ and $\mathrm{C} 4$ observed a bipolar signature in $B_{z}$ and peaks in $B_{x}$ and $|\boldsymbol{B}|$. The observed signatures are consistent with the passage of an Earthward-moving flux rope for $\mathrm{C} 2$ and $\mathrm{C} 3$, and an Earthward-moving travelling compression region (TCR) (Slavin et al., 2005) for C1 and C4.

The flux rope signatures are all of short duration (between 9 and $20 \mathrm{~s}$ ), therefore high-time resolution data are 

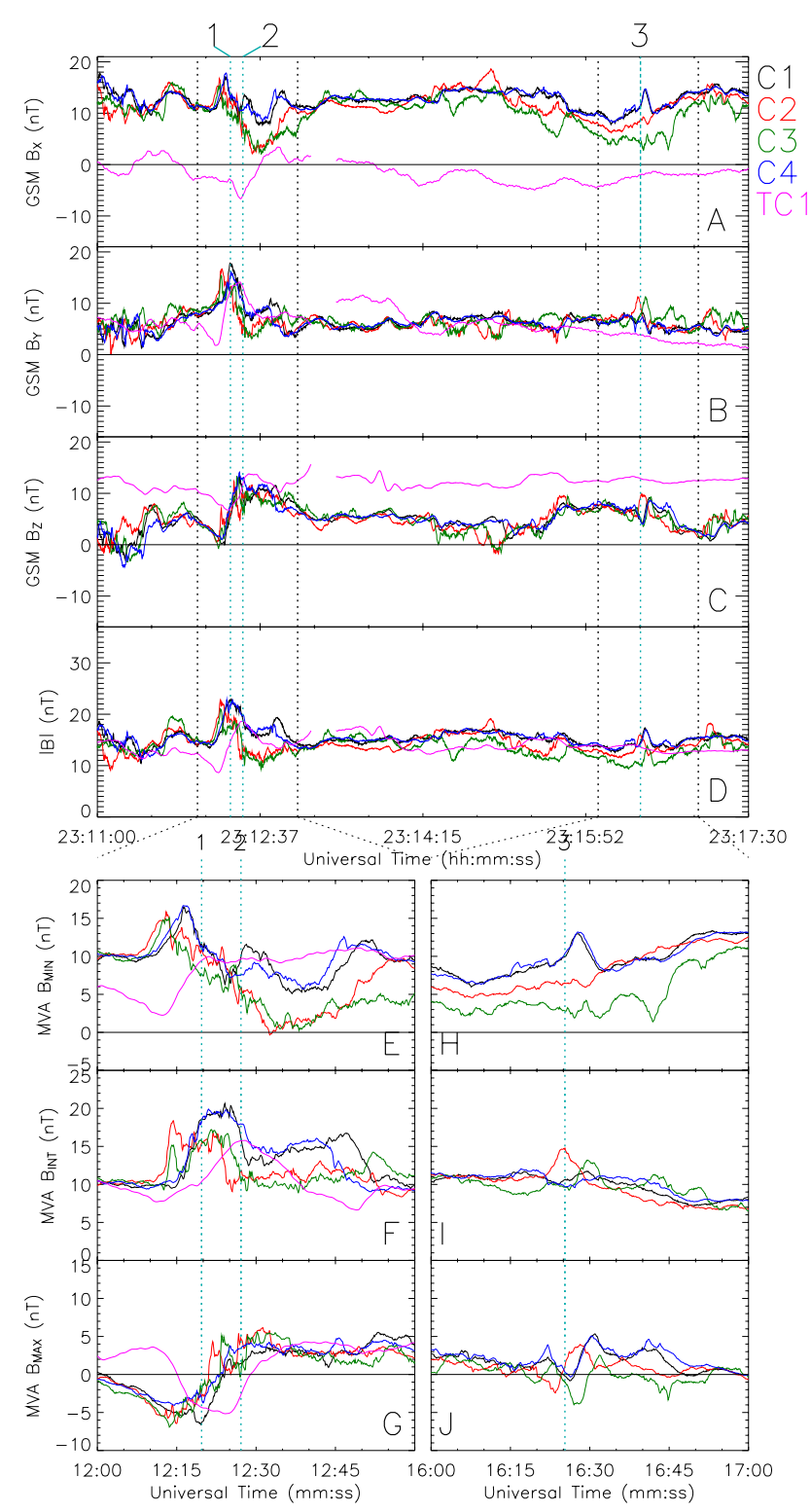

Fig. 2. Flux rope signatures for all four Cluster spacecraft and TC-1 (trace colors as for Fig. 1, panel D). Panels (A-C) show the three GSM components of the magnetic field, and panel (D) the magnitude of the magnetic field. Panels (E-G) show Signatures 1 and 2 in minimum variance coordinates. Panels (H-J) show Signature 3 in minimum variance coordinates. The Cluster data in Panels (E-J) are in a minimum variance coordinate frame calculated from $\mathrm{C} 2 \&$ Signature 3, while the TC-1 data are in a coordinate frame calculated from Signature 2.

required for accurate MVA. While Cluster FGM has satisfactory time resolution, because of contamination from the spacecraft magnetic field, only spin (4s) resolution FGM

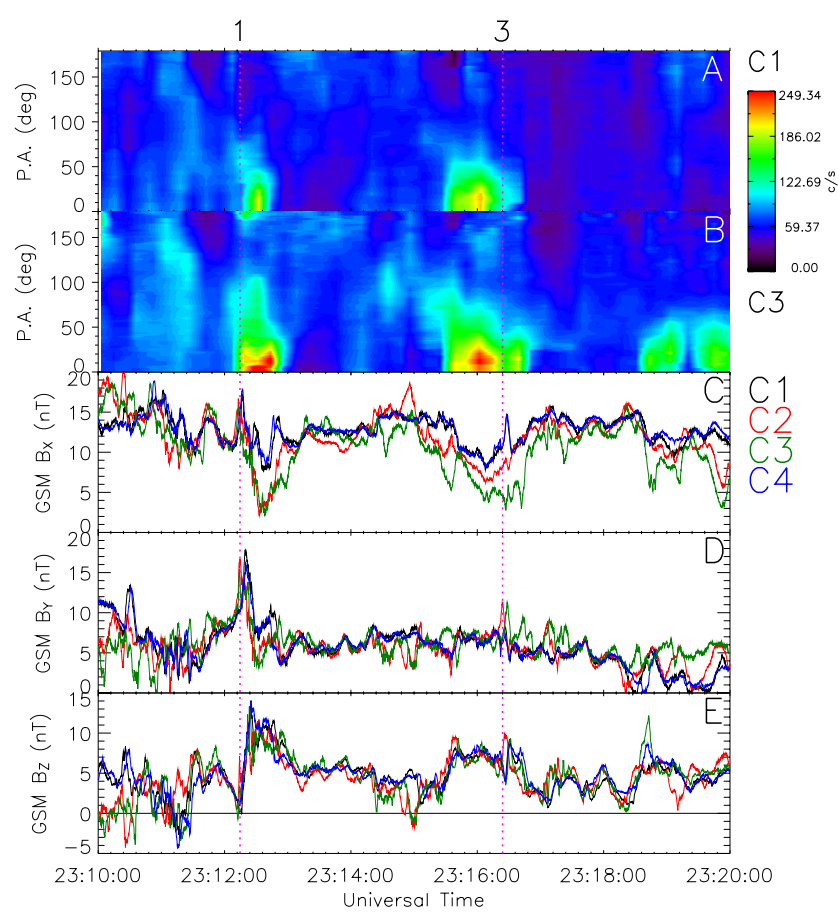

Fig. 3. Panels (A) and (B) show pitch angle-time spectrograms for HIA on board C1 and C3, respectively, and panels (C-E) GSM components of the magnetic field for all 4 Cluster spacecraft (colors as before). Signatures 1 and 3 are marked by magenta dotted lines.

data is normally available for TC-1. In order to provide a high enough number of data points for effective MVA, a 4$\mathrm{s}$ sliding window average was applied to the raw magnetic field data, removing any high frequency components, including much of the contamination. A spin axis offset correction was then applied to ensure that the time series conformed to the spin-resolution data values.

\subsection{Ion beam observations}

Figure 3 shows ion pitch angle data from the CIS-HIA instruments on board $\mathrm{C} 1$ and $\mathrm{C} 3$ (panels a and $\mathrm{b}$, respectively) and the three components of $\boldsymbol{B}$ for $\mathrm{C} 1-\mathrm{C} 4$ (panels c-e). Signatures 1 and 3 are marked here with magenta dotted lines. Earthward-directed, field-aligned ion beams were observed just after the encounter with Signature 1, and before and after Signature 3. These ion beams were detected in energy bins above $\sim 4 \mathrm{keV}$ and are accompanied by a weakening of $B_{x}$ and a strengthening of $B_{z}$. The beams are stronger and of longer duration at $\mathrm{C} 3$ than $\mathrm{C} 1$ and show no evidence of velocity dispersion. Similar ion features are seen at C3 at 23:19 and 23:20 UT, however there is no indication in the magnetic field data of any flux rope-like structures. No such ion features are seen in the TC- 1 data, around the time of Signature 2 or otherwise. 


\section{Data analysis}

The observations of Signatures 1 and 2 may either be interpreted as a single flux rope seen at Cluster then TC-1 in quick succession, or two individual flux ropes existing in the tail simultaneously. For one flux rope to be seen at two spacecraft, separated by $6.3 R_{E}$, within a few seconds, it has to be orientated in such a way that its propagation velocity allows it to cross each spacecraft at the correct time. It is essential, therefore, to confidently determine the axis orientation of each flux rope signature.

\subsection{Flux rope modelling}

While MVA has been used to successfully obtain an estimate of the orientation of a flux rope axis (e.g. Eastwood et al., 2002), fitting magnetic field data to a specific flux rope model often provides a more comprehensive result (e.g. Mulligan et al., 2001). Here we will use a simplified version of the cylindrically symmetric flux rope model described by Mulligan and Russell (2001) to determine the orientations of each flux rope signature, and hence whether the data represent more than one flux rope. This model was chosen because it does not assume flux ropes to be in a force-free state and Henderson et al. (2006) reported flux ropes seen in the Cluster dataset that were not force free. Three vector components of magnetic field are modelled as follows:

$$
\begin{aligned}
& B_{x}=B_{p} \frac{z}{R}\left[1-e^{-\left(\frac{R}{\sigma_{p} l}\right)^{n}}\right] \\
& B_{y}=B_{a} e^{-\left(\frac{R}{\sigma_{a} l}\right)^{m}} \\
& B_{z}=-B_{p} \frac{x}{R}\left[1-e^{-\left(\frac{R}{\sigma_{p} l}\right)^{n}}\right]
\end{aligned}
$$

where $B_{a}$ and $B_{p}$ represent axial and poloidal magnetic fields, respectively; $R$ is the distance to the flux rope axis; $l$ is the radius of the flux rope and $\sigma_{a}, \sigma_{p}, m$ and $n$ are dimensionless parameters. $x$ and $z$ represent the spacecraft position within the flux rope, and are generated from an impact parameter. In this coordinate system, the $y$ direction points along the flux rope axis, $x$ along the spacecraft trajectory and $z$ completes the right handed set. Note that there is no boundary condition setting the axial field to $0 \mathrm{nT}$ at the flux rope edge. Two rotation angles, $\theta$ and $\rho$ (rotations about the model $x$ and $z$ axes, respectively, applied to the model field), as well as $B_{a}, B_{p}, \sigma_{a}, \sigma_{p}, m, n$ and the impact parameter must be optimised, then, in order to determine the orientation of a flux rope axis. This fit is obtained through minimisation of the response function

$$
\begin{aligned}
B_{\text {res }}^{2}= & \left(B_{x^{\prime} \text { data }}-B_{x^{\prime} \text { model }}\right)^{2}+\left(B_{y^{\prime} \text { data }}-B_{y^{\prime} \text { model }}\right)^{2} \\
& +\left(B_{z^{\prime} \text { data }}-B_{z^{\prime} \text { model }}\right)^{2}
\end{aligned}
$$

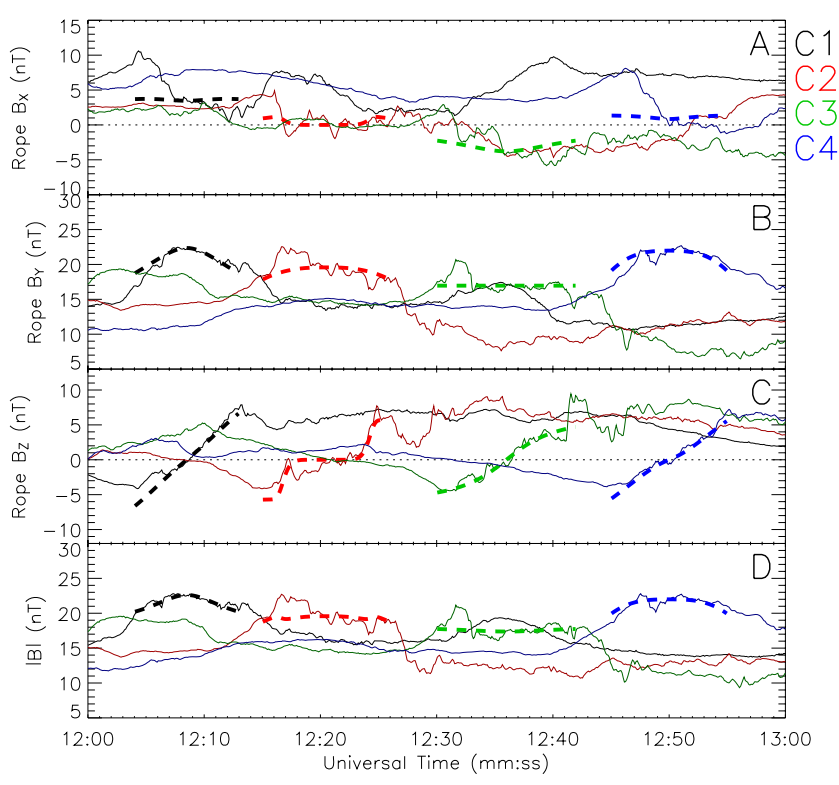

Fig. 4. Model fits for Signature 1. The thick dashed lines represent the model flux ropes, the thinner solid lines the FGM data. Colours as before. Panels (A-C) show the three field components in the flux rope model frame and panel (D) the total field. Data have been offset by $-12,3,18$ and 30 s for $\mathrm{C} 1, \mathrm{C} 2, \mathrm{C} 3$ and $\mathrm{C} 4$, respectively.

where ( $\left.B_{x^{\prime} \text { model }}, B_{y^{\prime} \text { model }}, B_{z^{\prime} \text { model }}\right)$ represents the model field rotated into GSM coordinates using $\theta$ and $\rho$, $\left(B_{x^{\prime} \text { data }}, B_{y^{\prime} \text { data }}, B_{z^{\prime} \text { data }}\right)$ the data from FGM and $B_{r e s}$ the residuals.

Figures 4, 5 and 6 show the model fits for Signatures 1, 2 and 3 , respectively. Panels $(a-c)$ in each figure show the three components of the magnetic field in the flux rope model frame, and panel (d) the field magnitude. The solid lines represent the FGM data and the dashed lines the model fits. For the purposes of fitting the model, the edges of each flux rope signature in the data were defined as the points of inflection in the bipolar signature seen in GSM $B_{z}$. In Fig. 4 data from $\mathrm{C} 1, \mathrm{C} 2, \mathrm{C} 3$ and $\mathrm{C} 4$ are time shifted for clarity by $-12,3,18$ and $30 \mathrm{~s}$, respectively. In the cases of $\mathrm{C} 1$ and $\mathrm{C} 4$, the model fit underestimates the contribution of GSM $B_{x}$ to the total field while overestimating the contribution made by GSM $B_{y}$ during the early part of the rope interval. If the data are rotated into the model frame, however, the majority of this discrepancy becomes confined to $B_{x}$ component, i.e. along the spacecraft trajectory. Since the model assumes cylindrical symmetry, any discrepancies between the model flux ropes and magnetic field data might be explained by a lack of cylindrical symmetry, which could be caused by "collisions" between the flux ropes and the plasma and magnetic fields ahead of them distorting the flux ropes from an axially symmetric shape (Slavin et al., 2003a,b). In Fig. 5 it is again the GSM $B_{x}$ component of the model field which fits the data 


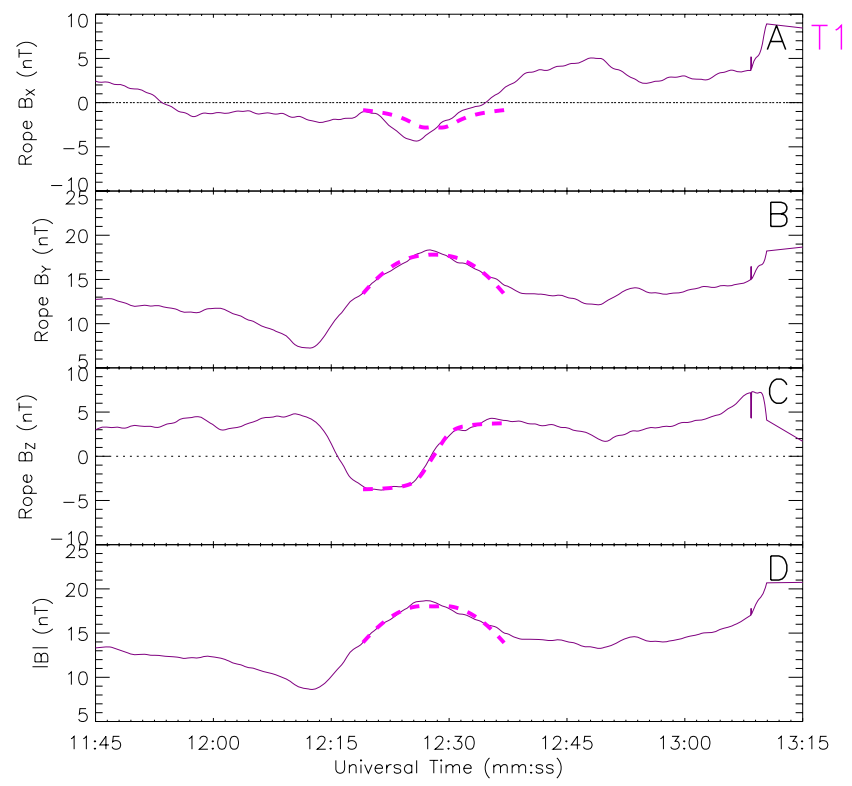

Fig. 5. Model fit for Signature 2. The thick dashed line represents the model flux rope, the thinner solid line the FGM data from TC1. Panels (A-C) show the three field components in the flux rope model frame and panel (D) the total field.

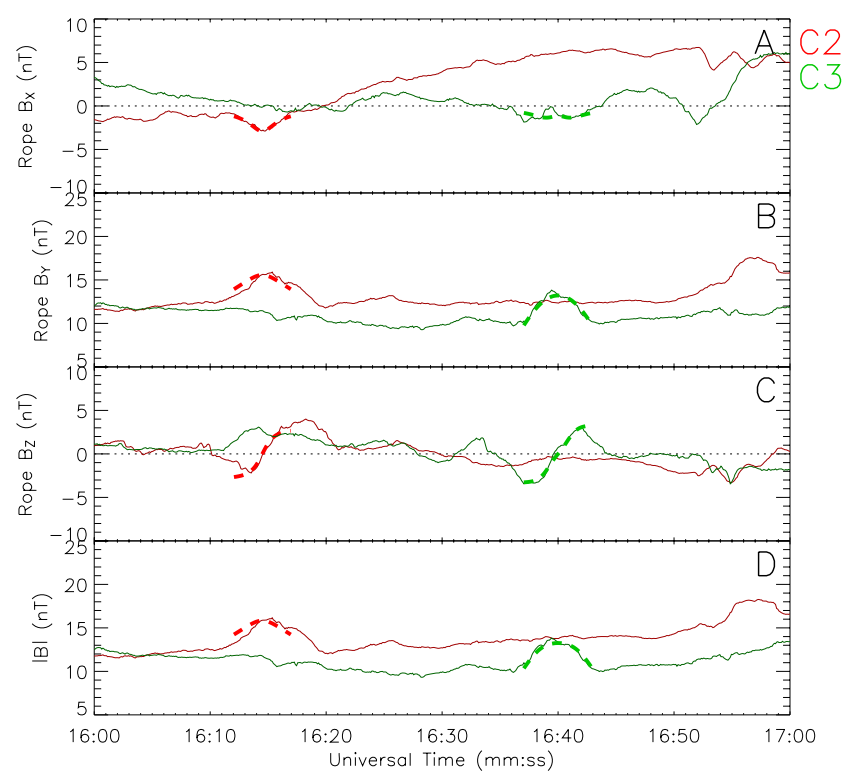

Fig. 6. Model fits for Signature 3. The thick dashed lines represent the model flux ropes, the thinner solid lines the FGM data. Colours as before. Panels (A-C) show the three field components in the flux rope model frame and panel (D) the total field. Data have been offset by -10 and 10 s for $\mathrm{C} 2$ and $\mathrm{C} 3$, respectively.

least well. In Fig. 6, the traces for $\mathrm{C} 2$ and $\mathrm{C} 3$ are offset by $\mp 10 \mathrm{~s}$, respectively.
Table 1 lists the axial and poloidal field strengths, rotation angles, impact parameters and radii returned from the model fits, as well as the estimated magnetic flux through the flux rope and the angle between the model axis and the MVA axis discussed above, for the spacecraft relevant to each signature. The impact parameters and radii were initially returned in time units, calculated from the spacecraft travel time through the flux rope signature (cf. Khurana et al., 1995), before being converted to kilometres by multiplying by the ion velocity $\left(\boldsymbol{v}_{\perp}\right)$ as projected onto the plane perpendicular to the flux rope axis. This should eliminate any overestimation of radii and impact parameters from spacecraft trajectories not perpendicular to the rope axis, which are not considered in the model equations. Any changes in magnetic field behaviour along a flux rope axis are assumed to be negligible on the scale of these observations.

The model fits for Signature 1 return rotation angles for the four Cluster spacecraft that are mutually consistent to within $7^{\circ}$ and $22^{\circ}$ for $\theta$ and $\rho$, respectively. This consistency increases confidence that the modelled rotation angles are correct, despite the differences in the magnetic field observed by each spacecraft. Furthermore, the impact parameters returned are largely consistent with the separation of the Cluster tetrahedron, as projected onto the plane perpendicular to the flux rope axis. In the case of Signature 3, the angles are also consistent between the $\mathrm{C} 2$ and $\mathrm{C} 3$, however the difference in impact parameters between the two spacecraft is too small when compared with the separation of the Cluster tetrahedron, and therefore the flux rope radii and enclosed magnetic fluxes calculated for Signature 3 are likely to be underestimates.

The difference in enclosed flux between Signature 3 $(\sim 10 \mathrm{kWb})$ and Signatures 1 and $2(\sim 60-80 \mathrm{kWb})$ might, then, be a result of this underestimate, rather than Signature 3 representing a much smaller flux rope. Again, a departure from cylindrical symmetry could explain this underestimate. In comparison with the tens of $\mathrm{kWb}$ enclosed by the flux ropes, which are expected to either be connected at both ends to the Earth, or to the IMF in the magnetosheath, a typical substorm can close several tenths of $\mathrm{GWb}$ of open lobe flux (Milan et al., 2004).

\subsection{Flux rope axis orientations}

Assuming a rope orientation perpendicular to the line connecting the spacecraft, for Signatures 1 and 2 to represent one flux rope, velocities of between $\sim 2900$ and $\sim 5700 \mathrm{~km} \mathrm{~s}^{-1}$ would be required. This figure is inconsistent with the velocity of propagation from 4-spacecraft timing at Cluster $\left(146 \mathrm{~km} \mathrm{~s}^{-1}\right)$ and data from the ion instruments on board the Cluster and TC-1 spacecraft; all of which give ion velocities of the order of $200 \mathrm{~km} \mathrm{~s}^{-1}$. If the rope axes were oriented along, or close to, the line connecting the spacecraft then a slower propagation velocity might be sufficient for one flux 
Table 1. Physical parameters returned from the flux rope model fits and the derived magnetic flux through each flux rope. $B_{a}$ and $B_{p}$ are the axial and poloidal fields, respectively; $\theta$ is the rotation angle about the model coordinate system $x$ axis; $\rho$ is the rotation angle about the model coordinate system $z$ axis; $z$ is the impact parameter; $l$ the flux rope radius and $\Phi$ the magnetic flux through the rope. $\alpha$ is the angle between the flux rope axis returned from MVA and the axis returned from the model fit. Quantities are rounded to the nearest nT, ${ }^{\circ}, \mathrm{km}$ or $\mathrm{kWb}$ as appropriate. A positive (negative) impact parameter denotes that the spacecraft passed above (below) the flux rope axis.

\begin{tabular}{lcccccccc}
\hline S/C & $B_{a}(\mathrm{nT})$ & $B_{p}(\mathrm{nT})$ & $\theta\left(^{\circ}\right)$ & $\rho\left(^{\circ}\right)$ & $z(\mathrm{~km})$ & $l(\mathrm{~km})$ & $\Phi(\mathrm{kWb})$ & $\alpha\left(^{\circ}\right)$ \\
\hline Signature 1 & & & & & & & & \\
C1 & 30 & 13 & 21 & -26 & 448 & 925 & 80 & 44 \\
C2 & 20 & 6 & 24 & -41 & 160 & 987 & 60 & 41 \\
C3 & 17 & 6 & 21 & -48 & -506 & 1177 & 74 & 57 \\
C4 & 22 & 12 & 28 & -36 & 214 & 922 & 59 & 53 \\
Signature 2 & & & & & & & & \\
TC1 & 18 & 8 & 45 & 5 & -343 & 1156 & 77 & 43 \\
Signature 3 & & & & & & & & \\
C2 & 17 & 3 & 37 & -42 & 185 & 463 & 13 & 33 \\
C3 & 13 & 3 & 38 & -14 & 117 & 523 & 10 & 7 \\
\hline
\end{tabular}

rope to be observed at both Cluster and TC-1 within a few seconds.

Figure 7 compares the axis orientations of each flux rope signature, as determined by the model fits on the magnetic field data from each individual spacecraft. Panel (a) of Fig. 7 shows the XY plane and panel (b) the YZ plane; the coloured squares represent the spacecraft positions at 23:12:30 UT, and the coloured lines the direction vector of the principal rope axis. The symbols representing Signature 3 have been offset by $(-1,4,-1) R_{E}$ for clarity. While the spacecraft positions are expressed in GSM coordinates, the vectors representing the flux rope axes use a coordinate system based on the orientation of the neutral sheet local to Cluster or TC-1. This coordinate system is defined by a rotation of GSM about the $X$ axis such that the new $X Z$ plane contains the normal to the tail neutral sheet, rather than the Earth's dipole axis. The normal to the neutral sheet was calculated once for the entire Cluster tetrahedron and separately for TC-1 by performing MVA on the closest possible neutral sheet crossing, by the correct spacecraft, to the flux rope encounter. For Signatures 1 and 3 this was a crossing by $\mathrm{C} 3$ as it left the thin current sheet region at 23:09:25 UT, 3 min before the encounter with Signature 1; for Signature 2 a TC-1 crossing at 23:11:50 UT, some $38 \mathrm{~s}$ before the flux rope encounter, was used. The neutral sheet normal was measured to be $\operatorname{GSM}(0.043,-0.063$, $0.997)$ at Cluster 3 and $\operatorname{GSM}(0.181,-0.484,0.856)$ at TC- 1 . This coordinate system was chosen because we believe that in a simple case any flux ropes present in the plasma sheet might lie in the plane of the current sheet. The angles between the plane containing neutral sheet normal and the flux rope axis orientation were calculated to be $72^{\circ}, 70^{\circ}, 72^{\circ}$ and $66^{\circ}$ for Cluster spacecraft $1-4$, respectively (Signature 1 ) and $74^{\circ}$ for TC-1 (Signature 2), all within $25^{\circ}$ of $90^{\circ}$. Comparing the Signature 2 (TC-1) flux rope axis with the Cluster neutral sheet normal gives an angle of $49^{\circ}$. Simulations by Winglee
(2004) have shown that shears in reconnection flows can also skew flux rope axes from an East-West orientation; however because all of the flux rope signatures are at a consistent angle to the plane containing the neutral sheet normal, despite different local orientations of the neutral sheet at Cluster and $\mathrm{TC}-1$, it can be inferred that flux rope orientation is linked to the tilt of the neutral sheet, at least in the north-south direction. There is no guarantee that the neutral sheet orientation remained the same between the crossing times and flux rope encounters, introducing an element of uncertainty to the calculated angles between rope axes and neutral sheet normals, as such the angles for Signature 3 were not calculated. The orientations of the flux rope axes are comparable to those observed by Slavin et al. (2003a) in their survey of flux ropes using the Geotail dataset, again increasing confidence in our axis orientations.

The Cluster and TC-1 rope axes lie approximately crosstail, the Cluster axes having a larger $\mathrm{x}$ component, pointing slightly towards TC-1. This tilt towards TC-1 means that a lower velocity than those calculated above (2900 $5700 \mathrm{~km} \mathrm{~s}^{-1}$ ) is needed for Signatures 1 and 2 to represent the same flux rope. Table 2 lists these velocities $\left(\boldsymbol{u}_{\perp}\right)$ calculated individually for each Cluster spacecraft. The perpendicular displacement $\left(\boldsymbol{R}_{\perp}\right)$ between each Signature 1 flux rope axis (assumed to be straight, and long enough) and TC1 were calculated and the plasma velocity as measured at Cluster projected onto this vector $\left(v_{\perp}^{\prime}\right)$. From these quantities, the time required $\left(\delta \mathrm{t}_{\perp}\right)$ for a flux rope to propagate from Cluster to TC-1 at $v_{\perp}^{\prime}$, the measured velocity, was calculated; for comparison, the velocity required $\left(\boldsymbol{u}_{\perp}\right)$ for a flux rope to propagate from Cluster to TC-1 in the observed time $(\delta \mathrm{t})$ was also calculated.

Despite the tilt towards TC-1, the required propagation velocities $\left(\boldsymbol{u}_{\perp}\right), 3687 \mathrm{~km} \mathrm{~s}^{-1}, 1664 \mathrm{~km} \mathrm{~s}^{-1}, 1223 \mathrm{~km} \mathrm{~s}^{-1}$ and $3511 \mathrm{~km} \mathrm{~s}^{-1}$ for $\mathrm{C} 1, \mathrm{C} 2, \mathrm{C} 3$ and $\mathrm{C} 4$, respectively, are still an 
Table 2. $\boldsymbol{R}_{\perp}$ is the perpendicular displacement between the Signature 1 flux rope axis and TC-1; $\boldsymbol{v}_{\perp} \perp$ is the observed plasma velocity as projected on to the displacement vector; $\delta \mathrm{t}$ is the observed difference between the time of flux rope encounter at the Cluster spacecraft and TC- $1 ; \delta \mathrm{t}_{\perp}$ is $\boldsymbol{R}_{\perp} / v^{\prime}{ }_{\perp}$, the required time difference between observation of Signature 1 and Signature 2 for a rope to propagate at the observed speed; and $\boldsymbol{u}_{\perp}{ }_{\perp}$ is the required propagation speed for a flux rope to travel from Cluster to TC- 1 in $\delta$ t. The measured velocities are too low to carry the Signature 1 flux rope to TC- 1 in the observed times.

\begin{tabular}{cccccc}
\hline $\mathrm{S} / \mathrm{C}$ & $\boldsymbol{R}_{\perp}(\mathrm{km})$ & $\boldsymbol{v}_{\perp}{ }^{\prime}\left(\mathrm{km} \mathrm{s}^{-1}\right)$ & $\boldsymbol{u}_{\perp}^{\prime}\left(\mathrm{km} \mathrm{s}^{-1}\right)$ & $\delta \mathrm{t}(\mathrm{s})$ & $\delta \mathrm{t}_{\perp}(\mathrm{s})$ \\
\hline $\mathrm{C} 1$ & 29,496 & 180 & 3687 & 8 & 164 \\
$\mathrm{C} 2$ & 21,640 & 177 & 1664 & 13 & 122 \\
$\mathrm{C} 3$ & 17,125 & 171 & 1223 & 14 & 100 \\
$\mathrm{C} 4$ & 24,579 & 179 & 3511 & 7 & 137 \\
\hline
\end{tabular}
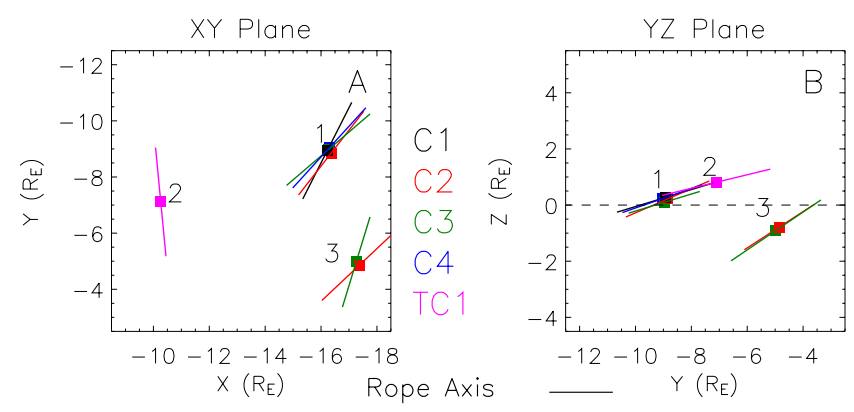

Fig. 7. A comparison of the various spacecraft positions (coloured squares - colours as for Figs. 1 and 2) and flux rope axis orientations (solid lines). Spacecraft positions are in GSM. The coordinate system used for flux rope axis directions is a rotation of GSM such that the XZ plane contains the normal to the tail current sheet, rather than the Earth's magnetic dipole axis. Signature 3 is offset by $(-1$, $4,-1) R_{E}$ for clarity.

order of magnitude greater than those measured at both Cluster and TC-1. Signatures 1 and 2, therefore cannot represent one straight flux rope. Given that the axes of Signatures 1 and 2 are of different orientations, do not lie along the line separating Cluster and TC-1, and their fields do not point in opposite directions; if the observations were to represent one flux rope, it would have to resemble a " $Z$ " shape. Magnetic tension forces would act to quickly straighten such a structure unless opposed by another force, however there is no evidence to suggest this is likely. Plasmoids observed in the magnetotail have been known to expand at rates as high as $\sim 100 \mathrm{kms}^{-1}$ as more reconnected flux is added to them (Slavin et al., 1999). Earthward moving flux ropes, however are unlikely to expand so rapidly, or may even contract, because of increased tail pressure closer to the Earth. Signatures 1 and 2, then, are unlikely to represent an expanding flux rope propagating over the spacecraft. This idea is also inconsistent with observed signature durations, plasma velocities and our model results, therefore we believe that the correct explanation is that Signatures 1 and 2, despite hav- ing similar flux content, represent two individual flux ropes existing in the tail at the same time.

\section{Discussion}

The observation of these simultaneously existing flux ropes in the near-Earth magnetotail, while novel in itself, can also be used to learn about the structure and dynamics of the tail during this event, particularly in the context of MXR. Our suggested interpretation of the data is that the flux ropes formed as part of a spatially extended system of competing multiple X-lines, encompassing the orbits of Cluster and TC1.

In Fig. 8 we suggest a sequence of events that explains the presence of these flux ropes in the magnetotail. Panel (a) shows the situation at 23:07 UT, when Cluster lies within the thin current sheet region (a notional current sheet is represented by light blue shading on the figure). The tailward flows detected by Cluster CIS are consistent with the presence of a reconnection site located Earthward of the spacecraft. The Earthward flows detected by TC-1 HIA at this time imply a reconnection site was located tailward of that spacecraft. It is impossible to unambiguously determine from the data the number of X-lines present between Cluster and TC1 at this time, however if a flux rope (later observed as Signature 2) was at that time extant, or being formed, between Cluster and TC-1 at least two reconnection sites would have to exist. By 23:09 UT, the time of substorm onset (panel b), Cluster had moved away from the neutral sheet towards the PSBL (consistent with the observed decrease in $\beta$ ) and thus did not observe any plasma flows. TC- 1 continued to observe moderate Earthward plasma flows, and we infer that the system of flux ropes was driven Earthward by fastest reconnection site that was part of the substorm. At 23:12 UT (panel c), Signature 1 had reached Cluster, and Signature 2 had reached TC-1. Upon leaving Signature 1, Cluster detected the first Earthward ion beam then returned to the PSBL. At 23:16 UT (panel d) Cluster detected the second Earthward ion beam then entered Signature 3, which was being driven Earthward by the continuing substorm reconnection. On leaving 
Signature 3, the third ion beam was detected and Cluster returned to the PSBL. The tailward directed plasma flows detected by TC-1 from 23:14 UT-23:21 UT are consistent with a reconnection site having moved Earthward past the spacecraft, again driven by the substorm. At 23:30 UT (panel e) Cluster returned to the central plasma sheet and crossed the neutral sheet, observing strong Earthward flows as it did so. This implies that the X-line driving the entire system remained tailward of Cluster throughout the interval. For three flux ropes to exist in the tail, a minimum of four $\mathrm{X}$-lines must exist. Figure 8 represents this minimum configuration, where the tailmost $\mathrm{X}$ line responsible for substorm expansion is one of those that forms Signature 3. Because none of these reconnection sites are directly observed by Cluster, however, a different X-line not associated with the observed flux ropes could instead be be responsible for the substorm expansion and driving the flux ropes Earthward. The significant difference between the amount of flux enclosed by each flux rope and the open flux closed in a substorm (Milan et al., 2004) implies that the dominant reconnection site in our system has to reconnect much more flux than those purely involved with the formation of the flux ropes. Unfortunately the location of that reconnection site and an explanation of its higher reconnection rate cannot be determined in this case.

During the passage of the flux rope labelled Signature 1, $B_{x}$ was seen to increase (Fig. 2, panel a). The spacecraft, therefore, sampled the upper portion of the flux rope. It is feasible then, and consistent with the short duration of the observed flows at the time of Signature 1 compared with those seen by TC-1 at Signature 2, that the tetrahedron was not ideally located to observe the flows associated with any reconnection, and it is only the passage of the flux rope creating a bulge in the plasma sheet that allowed the flowing plasma to be observed (Owen et al., 2005). Signature 3 can be interpreted as a more extreme example of the same idea. Here, two of the spacecraft ( $\mathrm{C} 2$ and $\mathrm{C} 3$ ) sample the very top of the flux rope (bipolar signature in $B_{z}$ and peak in $B_{y}$, while $\mathrm{C} 1$ and C4 miss the flux rope and instead see a TCR-like feature (bipolar signature in $B_{z}$ and peak in $B_{x}$ ). The configuration of the Cluster tetrahedron is consistent with this interpretation and it provides further evidence that the passage of flux ropes in the plasma sheet is indeed the source of TCRs in the lobe (Owen et al., 2005).

The observation of the Earthward-directed ion beams at Signatures 1 and 3 (Fig. 3, panels a and b) is consistent with this interpretation. Those field lines that were pushed into the path of the spacecraft by the flux ropes were perhaps connected to an X-Line (the source of the ion beams?) further downtail than Cluster, which is operating at both 23:12 UT and 23:16 UT. Since the beams show no evidence of velocity dispersion and no bidirectionality is observed, it is likely that the source of the ions is close to the spacecraft, rather than a distant neutral line. The observation of beams at the same energies at Signatures 1 and 3 implies that the source of the ions is operating steadily on the timescale of the event, and

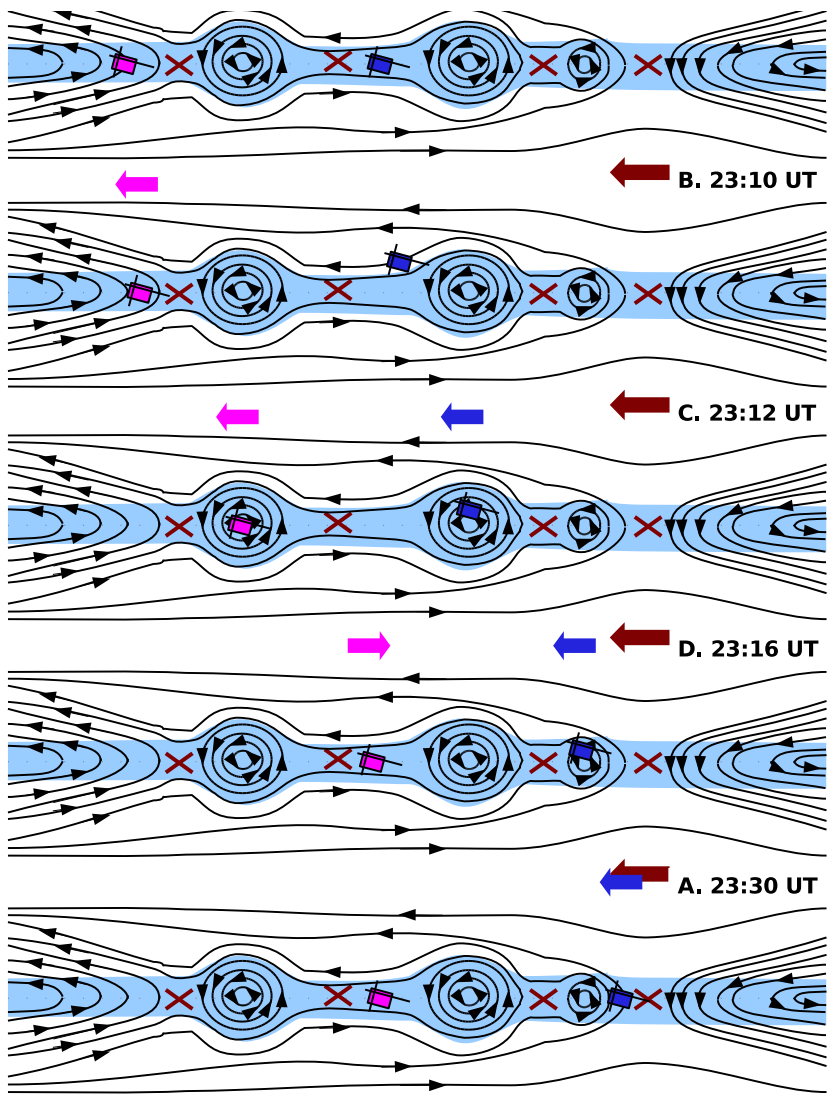

Fig. 8. An illustration of a possible sequence of events explaining the data. The dark blue spacecraft represents Cluster, and the magenta TC-1. Flows detected by each spacecraft are depicted as arrows of the relevant colour, whereas the red arrows represent the reconnection flow driving the system. Possible reconnection sites are marked as red Xs. A notional current sheet is depicted by the light blue shading. Panels (A-E) show the tail configuration at 23:07 UT, 23:10 UT, 23:12 UT, 23:16 UT and 23:30 UT, respectively. This figure represents the tail configuration with the minimum number of reconnection sites possible to explain the presence of three flux ropes.

is not a bursty phenomenon. The detection of the ions immediately after the flux rope at Signature 1, and then the switch off of the ions at 23:12:40 UT (C1) and 23:12:48 UT (C3) represent the spacecraft moving from the flux rope, through the trailing edge of the bulge caused by the passage of the flux rope, and into the relatively undisturbed plasma sheet between flux ropes. As $\mathrm{C} 1$ is further from the neutral sheet than C3, it observes the switch-off first, confirming the spatial nature of the feature. The switch on of ions at 23:15:35 UT (C1 and $\mathrm{C} 3$ ) then, indicate the spacecraft move onto the leading edge of the Signature 3 bulge. A dip in ion flux is observed by $\mathrm{C} 3$ as it moves through the flux rope before entering the trailing edge of the bulge. $\mathrm{C} 1$ sees no dip in ion flux, consistent with the spacecraft not entering the flux rope. Ion 
switch-off at 23:16:26 UT (C1) and 23:16:48 UT (C3) represent the spacecraft leaving the trailing edge of the bulge. No ion features associated with Signature 2 were observed by TC- 1 , consistent with our interpretation, since TC- 1 was located much deeper in the central plasma sheet than Cluster $\left(\beta \sim 2, \mathrm{~B}_{x} \sim 0\right)$.

The rotation and later dipolarization of the magnetic field at TC-1 is also consistent with the idea that there is an ongoing process working to restore the tail to a more relaxed configuration from its initial twisted state. This rotation and dipolarization may be two different processes working in tandem - the rotation of the field due to IMF clock angle changes and a slow dipolarization of the field due to steady (on the timescale of the event) reconnection further downtail. There is no evidence (e.g. sudden large dipolarizations) in the TC-1 magnetic field data for any bursty phenomena occurring further down tail.

It is interesting to note that no evidence of the Cluster flux ropes (Signatures 1 and 3) was seen by TC-1 later in the interval, so the spacecraft was either in the wrong place to see them, or they did not reach the position of TC-1. The length of flux ropes in their axial direction is impossible to determine using point measurements, so it feasible that the structures were not long enough to cross both Cluster and TC-1. It is also feasible that the Cluster flux ropes' motion stalled, or that they were absorbed into the surrounding magnetic field before reaching TC-1. Signature 2 was observed unusually close to the Earth for a flux rope (Slavin et al., 2003a), and the magnetic field measured at TC-1 became increasingly dipolar throughout the interval, increasing the likelihood of the Cluster flux ropes' re-reconnection with, and absorption into, the surrounding field before they reached TC-1.

The observations reported above add to a growing canon of observational and theoretical evidence that suggests the magnetotail can be significantly fragmented in three dimensions, and that simple 2-D models will no longer be sufficient to further our understanding of tail dynamics. Unfortunately data from the fortuitous conjunctions of spacecraft that have been available until now, including that presented here, are not always ideally suited for probing 3-D structures. The recently launched THEMIS mission, however, should provide many opportunities to study three dimensional phenomena in the tail in more detail.

\section{Conclusions}

We have presented an unusual observation of three flux ropes existing in the near-Earth magnetotail, two of which were observed simultaneously some $6.3 R_{E}$ apart. The third flux rope was seen at two of the four Cluster spacecraft, the others seeing a feature resembling a TCR. These observations of multiple small flux ropes in the near-tail support the interpretation that TCRs in the near-tail are consequences of the presence of such flux ropes (Slavin et al., 2005). All the flux ropes were observed to have similar orientations with respect to the local neutral sheet, despite differences in local neutral sheet orientation, implying flux rope orientation is linked to neutral sheet tilt. Evidence suggests these flux ropes were formed through reconnection at a minimum of four competing X-points and were driven Earthward by the fastest reconnecting X point, located tailward of the Cluster tetrahedron.

Acknowledgements. The authors wish to thank the World Data Center for Geomagnetism, Kyoto for AE index data, the NASA CDAWeb for access to Geotail data and H. U. Frey for help with IMAGE data and software. A. P. Walsh and P. D. Henderson are supported by UK STFC studentship funding. R. J. Wilson was supported by the STFC-funded PEACE post-launch support grant. I. V. Alexeev is supported by the STFC-funded MSSL rolling grant.

Topical Editor I. A. Daglis thanks J. A. Slavin and two other anonymous referees for their help in evaluating this paper.

\section{References}

Baker, D. N., Pulkkinen, T. I., Angelopoulos, V., Baumjohann, W., and McPherron, R. L.: Neutral line model of substorms: Past results and present view, J. Geophys. Res., 101, 12 975-13010, doi:10.1029/95JA03753, 1996.

Balogh, A., Carr, C. M., Acuna, M. H., Dunlop, M. W., Beek, T. J., Brown, P., Fornaçon, K.-H., Georgescu, E., Glassmeier, K.-H., Harris, J., Musmann, G., Oddy, T., and Schwingenschuh, K.: The Cluster Magnetic Field Investigation: overview of in-flight performance and initial results, Ann. Geophys., 19, 1207-1217, 2001, http://www.ann-geophys.net/19/1207/2001/.

Carr, C., Brown, P., Zhang, T. L., Gloag, J., Horbury, T., Lucek, E., Magnes, W., O’Brien, H., Oddy, T., Auster, U., Austin, P., Aydogar, O., Balogh, A., Baumjohann, W., Beek, T., Eichelberger, H., Fornacon, K.-H., Georgescu, E., Glassmeier, K.-H., Ludlam, M., Nakamura, R., and Richter, I.: The Double Star magnetic field investigation: instrument design, performance and highlights of the first year's observations, Ann. Geophys., 23, 27132732, 2005, http://www.ann-geophys.net/23/2713/2005/.

Cowley, S. W. H.: Magnetospheric asymmetries associated with the y-component of the IMF, Planet. Space. Sci., 29, 79-96, doi: 10.1016/0032-0633(81)90141-0, 1981.

Eastwood, J. P., Balogh, A., Dunlop, M. W., and Smith, C. W.: Cluster observations of the heliospheric current sheet and an associated magnetic flux rope and comparisons with ACE, J. Geophys. Res., 107, 1365-1373, 2002.

Eastwood, J. P., Sibeck, D. G., Slavin, J. A., Goldstein, M. L., Lavraud, B., Sitnov, M., Imber, S., Balogh, A., Lucek, E. A., and Dandouras, I.: Observations of multiple X-line structure in the Earth's magnetotail current sheet: A Cluster case study, Geophys. Res. Lett., 32, 11 105, doi:10.1029/2005GL022509, 2005.

Fazakerley, A. N., Carter, P. J., Watson, G., Spencer, A., Sun, Y. Q., Coker, J., Coker, P., Kataria, D. O., Fontaine, D., Liu, Z. X., Gilbert, L., He, L., Lahiff, A. D., Mihaljcic, B., Szita, S., Taylor, M. G. G. T., Wilson, R. J., Dedieu, M., and Schwartz, S. J.: The Double Star Plasma Electron and Current Experiment, Ann. Geophys., 23, 2733-2756, 2005, http://www.ann-geophys.net/23/2733/2005/.

Frey, H. U. and Mende, S. B.: Substorm onsets as observed by IMAGE-FUV, Proc. 8th Int. Conf. Substorms, pp. 71-75, 2006. 
Henderson, P. D., Owen, C. J., Alexeev, I. V., Slavin, J., Fazakerley, A. N., Lucek, E., and Reme, H.: Cluster observations of flux rope structures in the near tail, Ann. Geophys., 24, 651-666, 2006, http://www.ann-geophys.net/24/651/2006/.

Hughes, W. J. and Sibeck, D. G.: On the 3-dimensional structure of plasmoids, Geophys. Res. Lett., 14, 636-639, 1987.

Johnstone, A. D., Alsop, C., Burge, S., Carter, P. J., Coates, A. J., Coker, A. J., Fazakerley, A. N., Grande, M., Gowen, R. A., Gurgiolo, C., Hancock, B. K., Narheim, B., Preece, A., Sheather, P. H., Winningham, J. D., and Woodliffe, R. D.: Peace: a Plasma Electron and Current Experiment, Space Sci. Rev., 79, 351-398, 1997.

Khurana, K. K., Kivelson, M. G., Frank, L. A., and Paterson, W. R.: Observations of magnetic flux ropes and associated currents in Earth's magnetotail with the Galileo spacecraft, Geophys. Res. Lett., 22, 2087-2090, doi:10.1029/95GL01518, 1995.

Lepping, R. P., Jones, J. A., and Burlaga, L. F.: Magnetic Field Structure of Interplanetary Magnetic Clouds at 1 AU, J. Geophys. Res., 95, 11 957-11 965, 1990.

Mende, S. B., Heetderks, H., Frey, H. U., Lampton, M., Geller, S. P., Habraken, S., Renotte, E., Jamar, C., Rochus, P., Spann, J., Fuselier, S. A., Gerard, J.-C., Gladstone, R., Murphree, S., and Cogger, L.: Far ultraviolet imaging from the IMAGE spacecraft. 1. System design, Space Sci. Rev., 91, 243-270, 2000.

Milan, S. E., Cowley, S. W. H., Lester, M., Wright, D. M., Slavin, J. A., Fillingim, M., Carlson, C. W., and Singer, H. J.: Response of the magnetotail to changes in the open flux content of the magnetosphere, Journal of Geophysical Research (Space Physics), 109, A04220, doi:10.1029/2003JA010350, 2004.

Mulligan, T. and Russell, C. T.: Multispacecraft modeling of the flux rope structure of interplanetary coronal mass ejections: Cylindrically symmetric versus nonsymmetric topologies, J. Geophys. Res., 106, 10 581-10 596, doi:10.1029/ 2000JA900170, 2001.

Mulligan, T., Russell, C. T., Anderson, B. J., and Acuna, M. H.: Multiple spacecraft flux rope modeling of the Bastille Day magnetic cloud, Geophys. Res. Lett., 28, 4417-4420, doi:10.1029/ 2001GL013293, 2001.

Nakamura, R., Baumjohann, W., Zhang, T. L., Carr, C. M., Balogh, A., Fornacon, K.-H., Georgescu, E., Rème, H., Dandouras, I., Takada, T., Volwerk, M., Asano, Y., Runov, A., Eichelberger, H., Klecker, B., Mouikis, C., Kistler, L. M., and Amm, O.: Cluster and Double Star observations of dipolarization, Ann. Geophys., 23, 2915-2920, 2005, http://www.anngeophys.net/23/2915/2005/.

Ogino, T., Walker, R. J., and Ashour-Abdalla, M.: Magnetic flux ropes in 3-dimensional MHD simulations, Washington DC American Geophysical Union Geophysical Monograph Series, pp. 669-678, 1990

Owen, C. J., Slavin, J. A., Fazakerley, A. N., Dunlop, M. W., and Balogh, A.: Cluster electron observations of the separatrix layer during traveling compression regions, Geophys. Res. Lett., 32, L03104, doi:10.1029/2004GL021420, 2005.

Rème, H., Aoustin, C., Bosqued, J. M., Dandouras, I., Lavraud, B., Sauvaud, J. A., Barthe, A., Bouyssou, J., Camus, T., CoeurJoly, O., Cros, A., Cuvilo, J., Ducay, F., Garbarowitz, Y., Medale, J. L., Penou, E., Perrier, H., Romefort, D., Rouzaud, J., Vallat, C., Alcayd, D., Jacquey, C., Mazelle, C., d'Uston, C., Mobius, E., Kistler, L. M., Crocker, K., Granoff, M., Mouikis, C.,
Popecki, M., Vosbury, M., Klecker, B., Hovestadt, D., Kucharek, H., Kuenneth, E., Paschmann, G., Scholer, M., Sckopke, N., Seidenschwang, E., Carlson, C. W., Curtis, D. W., Ingraham, C., Lin, R. P., McFadden, J. P., Parks, G. K., Phan, T., Formisano, V., Amata, E., Bavassano-Cattaneo, M. B., Baldetti, P., Bruno, R., Chionchio, G., Lellis, A. D., Marcucci, M. F., Pallocchia, G., Korth, A., Daly, P. W., Graeve, B., Rosenbauer, H., Vasyliunas, V., McCarthy, M., Wilber, M., Eliasson, L., Lundin, R., Olsen, S., Shelley, E. G., Fuselier, S., Ghielmetti, A. G., Lennartsson, W., Escoubet, C. P., Balsiger, H., Friedel, R., Cao, J.-B., Kovrazhkin, R. A., Papamastorakis, I., Pellat, R., Scudder, J., and Sonnerup, B.: First multispacecraft ion measurements in and near the Earth's magnetosphere with the identical Cluster ion spectrometry (CIS) experiment, Ann. Geophys., 19, 1303-1354, 2001, http://www.ann-geophys.net/19/1303/2001/.

Rème, H., Dandouras, I., Aoustin, C., Bosqued, J. M., Sauvaud, J. A., Vallat, C., Escoubet, P., Cao, J. B., Shi, J., BavassanoCattaneo, M. B., Parks, G. K., Carlson, C. W., Pu, Z., Klecker, B., Moebius, E., Kistler, L., Korth, A., Lundin, R., and The Hia Team: The HIA instrument on board the Tan Ce 1 Double Star near-equatorial spacecraft and its first results, Ann. Geophys., 23 , 2757-2774, 2005, http://www.ann-geophys.net/23/2757/2005/.

Schindler, K.: A Theory of the Substorm Mechanism, J. Geophys. Res., 79, 2803-2810, 1974.

Slavin, J. A., Hesse, M., Owen, C. J., Taguchi, S., Fairfield, D. H., Lepping, R. P., Kokubun, S., Mukai, T., Lui, A. T. Y., Anderson, R. R., Matsumoto, H., and Sutcliffe, P. R.: Dual spacecraft observations of lobe magnetic field perturbations before, during and after plasmoid release, Geophys. Res. Lett., 26, 2897-2900, doi:10.1029/1999GL003606, 1999

Slavin, J. A., Lepping, R. P., Gjerloev, J., Fairfield, D. H., Hesse, M., Owen, C. J., Moldwin, M. B., Nagai, T., Ieda, A., and Mukai, T.: Geotail observations of magnetic flux ropes in the plasma sheet, J. Geophys. Res., 108, 1015-1032, 2003a.

Slavin, J. A., Lepping, R. P., Gjerloev, J., Goldstein, M. L., Fairfield, D. H., Acuna, M. H., Balogh, A., Dunlop, M., Kivelson, M. G., Khurana, K., Fazakerley, A., Owen, C. J., Reme, H., and Bosqued, J. M.: Cluster electric current density measurements within a magnetic flux rope in the plasma sheet, Geophys. Res. Lett., 30, 1362-1365, 2003b.

Slavin, J. A., Transkanen, E. I., Hesse, M., Owen, C. J., Dunlop, M. W., Imber, S., Lucek, E. A., Balogh, A., and Glassmier, K.-H.: Cluster observations of traveling compression regions in the near-tail, J. Geophys. Res., 110, A06207, doi:10.1029/2004JA010878, 2005.

Sonnerup, B. U. O. and Cahill, L. J.: Magnetopause structure and attitude from Explorer 12 observations, J. Geophys. Res., 72, 171-183, 1967.

Winglee, R. M.: Ion cyclotron and heavy ion effects on reconnection in a global magnetotail, J. Geophys. Res., 109, A09206, doi: 10.1029/2004JA010385, 2004.

Xiao, C. J., Pu, Z. Y., Ma, Z. W., Fu, S. Y., Huang, Z. Y., and Zong, Q. G.: Inferring of flux rope orientation with the minimum variance analysis technique, J. Geophys. Res., 109, A11218, doi:10.1029/2004JA010594, 2004.

Zwingmann, W.: Self-consistent magnetotail theory - Equilibrium structures including arbitrary variation along the tail axis, J. Geophys. Res., 88, 9101-9108, 1983. 